\title{
Clinical Intervention Using Body Shadows for a Patient with Complex Regional Pain Syndrome Who Reported Severe Pain and Self-Disgust Toward the Affected Site: A Case Report
}

This article was published in the following Dove Press journal:

Journal of Pain Research

\author{
Yoshiyuki Hirakawa' \\ Akira Fujiwara ${ }^{2}$ \\ Ryota Imai (iD ${ }^{3,4}$ \\ Yuki Hiraga (iD) ${ }^{1,5}$ \\ Shu Morioka (iD) ${ }^{4,6}$ \\ 'Department of Rehabilitation, Fukuoka \\ Rehabilitation Hospital, Fukuoka City, \\ Fukuoka, Japan; ${ }^{2}$ Department of \\ Orthopedics, Fukuoka Reha Orthopedic \\ Clinic, Fukuoka City, Fukuoka, Japan; \\ ${ }^{3}$ School of Rehabilitation, Osaka \\ Kawasaki Rehabilitation University, \\ Kaizuka City, Osaka, Japan; ${ }^{4}$ Department \\ of Occupational Therapy, International \\ Univesrity of Health and Welfare, Okawa, \\ Fukuoka, Japan; ${ }^{5}$ Neurorehabilitation \\ Research Centre, Kio University, Nara, \\ City, Nara, Japan; ${ }^{6}$ Department of \\ Neurorehabilitation, Graduate School of \\ Health Sciences, Kio University, Nara \\ City, Nara, Japan
}

\begin{abstract}
A woman in her thirties developed complex regional pain syndrome in her left shoulder due to a traffic accident. She demonstrated autonomic nervous symptoms (swelling, sweating, and skin color asymmetry) in her left hand, severe allodynia, neglect-like symptoms (NLS), impaired body image associated with impaired body awareness, and functional impairment of the left shoulder and elbow. She also reported physical self-disgust toward her affected limb, describing it as "reptilian," as well as aversion to touching others; this body awareness exacerbated her pain and NLS. We therefore conducted stepwise interventions using body shadows. The intervention did not trigger physical self-disgust, enabling formation of body ownership and a body image unaccompanied by pain. Consequently, the patient showed improvements in pain, NLS, and autonomic nervous symptoms.
\end{abstract}

Keywords: pain, neglect-like symptoms, physical self-disgust, body shadow intervention, body image

\section{Introduction}

In type I complex regional pain syndrome (CRPS), pain is caused and modulated by changes in the peripheral nervous system (especially inflammation and autonomic neuropathy ${ }^{1}$ as well as malnutrition) and plastic changes in the central nervous system. ${ }^{2}$ These changes may be responsible for the diminished perception associated with shrinkage of the somatosensory area corresponding to the affected $\operatorname{site}^{3-10}$ as well as dysfunction of the inferior parietal lobule, which governs multisensory integration. ${ }^{11,12}$ In addition, the absence of accurate sensory feedback results in incongruence between movement and sensory feedback. The persistence of this sensory discrepancy and the occurrence of motor dysfunction in the affected $\operatorname{limb}^{13}$ have been shown to exacerbate pain. $^{14-16}$ Neglect-like symptoms (NLS) may arise through a plastic process in this sensorimotor representation. ${ }^{17}$ There are two types of NLS: cognitive neglect $(\mathrm{CN})$, wherein patients have a diminished sense of ownership (SoO) of their own limb (ie, the patients feel that their limb is not their own), and motor neglect (MN), wherein patients require particular attention and effort to move their own limbs or possess a diminished sense of agency (SOA). SOA refers to the subjective awareness of initiating and controlling one's own action. These two symptoms are reported to be present in $47 \%$ of patients with CRPS, ${ }^{18,19}$ and various treatments have been developed to reduce these
Correspondence: Yoshiyuki Hirakawa Department of Rehabilitation, Fukuoka Rehabilitation Hospital, 7-770 Nokata, Nishi-ku, Fukuoka City 819-855I,

Fukuoka, Japan

Tel +8I $92812-1555$

Fax +8I $9281 \mathrm{I}-0330$

Email yutsuki0903@yahoo.co.jp 
symptoms. For example, mirror therapy (MT) aims to correct the incongruency between motion and perception and to encourage the formation of a body image, ${ }^{20}$ while graded motor imagery attempts to integrate vision and kinesthesia gradually. $^{21-23}$

We conducted rehabilitation for a patient with CRPS who had NLS. The patient demonstrated a reduced SoO due to pain and sensory abnormality in the affected site. The initial rehabilitation intervention used imaged movement and MT. Pain was alleviated only during MT; however, this effect did not last.

Body shadows have been recently studied in relation to construction of the body images. Body shadows are projections of hands and other objects onto walls and other surfaces by light sources. These shadows are considered to direct attention to one's own body, ${ }^{24}$ improve body perception, and contribute to the construction of the body image. ${ }^{25}$ Although body shadows are merely abstract representations of the body, they can trigger perception of movement. We conducted stepwise interventions using body shadows, hypothesizing that the patient who demonstrated physical self-disgust of their own limb may form a body image through body shadows, which do not trigger physical self-disgust.

\section{Case}

A woman in her thirties experienced a left labrum tear due to a traffic accident. Despite undergoing labrum repair surgery, she continued to have pain and functional impairment, and she was diagnosed with CRPS. Six years later, she was referred to a hospital for pain alleviation and improvement in functional impairment. She then began twice-weekly outpatient rehabilitation. The patient provided written informed consent for the publication of this case report, including images. Approval was obtained from the Fukuoka Rehabilitation Hospital Institutional Review Board (approval no.: FRH2018-R-021).

\section{Early Assessment}

During early assessments (Table 1), the patient demonstrated moderate allodynia, sweating asymmetry, swelling, and cold sensation from her left shoulder to her fingers as well as a change in skin color asymmetry from her forearm to her fingers. She also presented with fixed dystonia, wherein she constantly held her forearm and hand in front of her chest with her fingers clenched. Additionally, she exhibited decreased range of motion and her left shoulder flexion was $90^{\circ}$. These symptoms met the Budapest criteria.
The patient's pain around her shoulder and in her forearm and hand was assessed as 8/10 on the 11-point numerical rating scale (NRS). NRS is a tool for obtaining subjective response as regards the degree of one's own pain on a scale from 0 (no pain at all) to 10 (unbearable pain). The patient demonstrated a high total score of 146/ 220 on the Japanese version of the Short-Form McGill Pain Questionnaire 2 (SF-MPQ-2). SF-MPQ-2 is a reliable multidimensional pain assessment tool comprising four sub-items - namely, continuous pain, intermittent pain, predominantly neuropathic pain, and affective descriptors. She demonstrated moderate to severe pain in all of her subscale scores (continuous pain, 44/60; intermittent pain, 42/60; neuropathic pain, 40/60; affective descriptors, 20/40). We assessed NLS using the questionnaire designed by Frettlöh et al. ${ }^{26}$ The five items of this questionnaire assess whether subjects can correctly recognize the presence of their own limb in $\mathrm{CN}$ and whether subjects pay any special attention when moving their own limb in MN. The patient's total NLS score was 480/500, her MN score was 290/300, and her CN score was 190/200, all indicating severe NLS. The patient's physical self-disgust toward her own limb was assessed with the NRS, wherein 0 indicated "no physical self-disgust" and 10 indicated "extremely strong physical self-disgust"; the patient rated as 10. Assessment of autonomic nervous symptoms revealed marked left-right differences in skin color change and swelling.

\section{Body Shadow Intervention First Step}

The purpose of this intervention was to allow the patient to form $\mathrm{SoO}$ and SoA, with the body shadow extending from her hand to the elbow. First, a cylindrical balloon was inserted into the left sleeve of a long-sleeve shirt to imitate the patient's left arm (Figure 1A). The patient did not pass her left arm through the sleeve but instead kept it inside the shirt (Figure 1B). The left hand was projected as a body shadow using the therapist's left hand (Figure 1C). As the patient observed this body shadow, she was instructed to repeatedly flex and extend the fingers on her right hand. The therapist concurrently flexed and extended the fingers on his left hand in synchronization with the patient's hand. The synchronization of the patient's motor intention and the visual feedback of the body shadow left hand created an SoA and SoO over the body shadow left hand. The patient did not complain of physical self-disgust or repulsion of the body shadow. 
Table I Changes in Evaluation Items

\begin{tabular}{|c|c|c|c|c|c|c|c|c|}
\hline & \multirow{2}{*}{$\begin{array}{l}6 \text { Months Pre-BS (Ist } \\
\text { Assessment) }\end{array}$} & \multirow{2}{*}{$\begin{array}{l}3 \text { Months } \\
\text { Pre-BS }\end{array}$} & \multirow{2}{*}{$\begin{array}{l}\text { I Day } \\
\text { Pre-BS }\end{array}$} & BS-Id & BS-8d & BS-13d & BS-32d & BS-48d \\
\hline & & & & BS-I & BS-5 & BS-7 & BS- 15 & BS-20 \\
\hline Pain (NRS) & 8 & 9 & 8 & 6 & 4 & 3 & 4 & 3 \\
\hline SF-MPQ-2 total & 146 & 145 & 130 & 112 & 84 & 79 & 68 & 54 \\
\hline SF-MPQ-2 (continuous pain) & 44 & 41 & 46 & 42 & 24 & 17 & 18 & 12 \\
\hline SF-MPQ-2 (intermittent pain) & 42 & 40 & 41 & 38 & 27 & 36 & 28 & 22 \\
\hline SF-MPQ-2 (neuropathic pain) & 40 & 42 & 33 & 30 & 33 & 26 & 22 & 20 \\
\hline SF-MPQ-2 (affective pain) & 20 & 22 & 10 & 2 & 0 & 0 & 0 & 0 \\
\hline NLS & 480 & 475 & & 285 & 205 & 150 & 140 & 125 \\
\hline NLS-MN & 290 & 285 & & 175 & 135 & 110 & 100 & 95 \\
\hline NLS-CN & 190 & 190 & & 110 & 70 & 40 & 40 & 30 \\
\hline Physical self-disgust & 9 & 10 & & 5 & 3 & 2 & 0 & 1 \\
\hline \multicolumn{9}{|l|}{$\begin{array}{l}\text { Autonomic nervous symptoms } \\
\text { (left-right difference) }\end{array}$} \\
\hline Skin color change & ++ & ++ & ++ & + & + & - & \pm & - \\
\hline Edema & ++ & ++ & ++ & + & + & - & - & - \\
\hline \multicolumn{9}{|l|}{ Rehabilitation program } \\
\hline Static stretch & \multicolumn{8}{|c|}{$\longrightarrow$} \\
\hline Muscle strength training & \multicolumn{8}{|c|}{$\longrightarrow$} \\
\hline Mirror therapy & \multicolumn{3}{|c|}{$\longrightarrow$} & & & & & \\
\hline Body shadow Ist step & & & & \multicolumn{5}{|c|}{$\longrightarrow$} \\
\hline \multicolumn{9}{|l|}{ Body shadow 2nd step } \\
\hline Body shadow 3rd step & & & & & & & & $\longrightarrow$ \\
\hline
\end{tabular}

Abbreviations: BS, body shadow; NRS, numerical rating scale; SF-MPQ-2, Short-Form McGill Pain Questionnaire 2; MN, motor neglect; CN, cognitive neglect; BS-I, body shadow first session; BS-Id, day I of the body shadow intervention.

\section{Second Step}

The objectives were to allow the patient to form SoO and SoA over a body shadow of her own hand and to simulate touching an object with her own hand. First, the body shadow of the imitation left arm and the therapist's hand was replaced with a body shadow of the patient's own left arm. The alleviation of physical self-disgust and repulsion achieved in the first step strengthened the patient's $\mathrm{SoO}$ and SoA over her body shadow; therefore, she was able to flex and extend her left fingers without any pain. The patient was gradually able to use her body shadow to "touch" her right hand and her face (Figure 1D) as well as another person's hand (Figure 1E). The patient said, "I feel like I'm really touching things but it doesn't hurt, and I don't feel scared." In other words, although she was not touching her own body, the intervention triggered the illusion of real touch. Thus, we were able to simulate the left hand touching other people and objects without pain or fear. In addition, the patient's physical self-disgust and repulsion toward her left arm gradually disappeared.

\section{Third Step}

The purpose was to extend the body shadow's effect on the patient's shoulder. First, a body shadow was projected by illuminating the patient from behind. The patient was instructed to perform right shoulder adduction and abduction while looking at this body shadow. The therapist performed adduction and abduction of the imitation left arm concurrently with the patient's motor intention. After confirming that pain was not exacerbated, we replaced the imitation left arm with the patient's own left arm (Figure 1F). This exercise expanded the patient's $\mathrm{SoO}$ and SoA from her left hand to her entire left arm and enabled her to move her left arm without the fear of pain. 


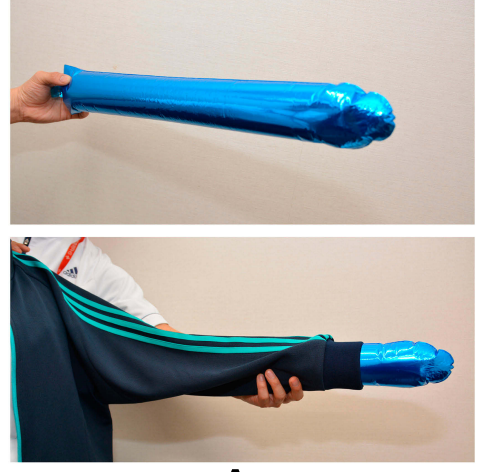

A

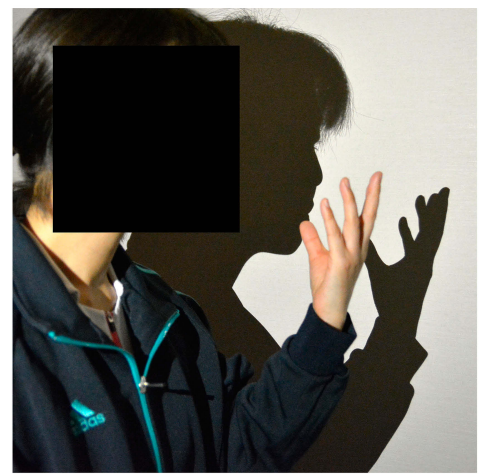

D

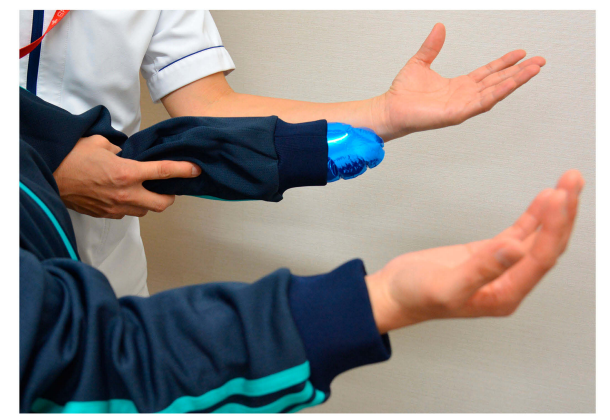

B

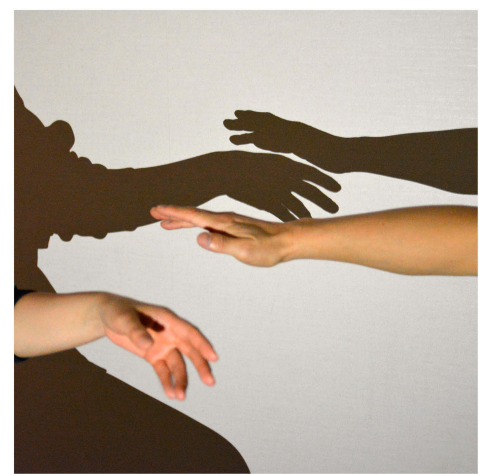

E

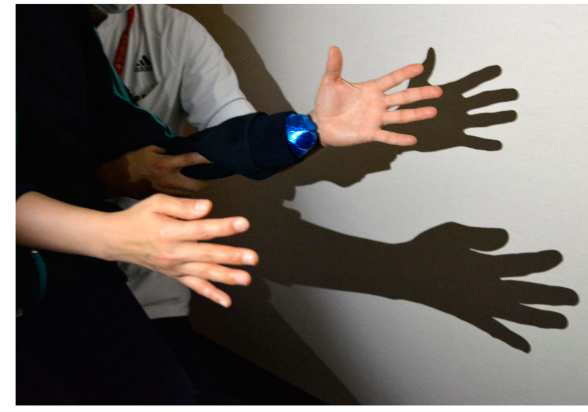

C

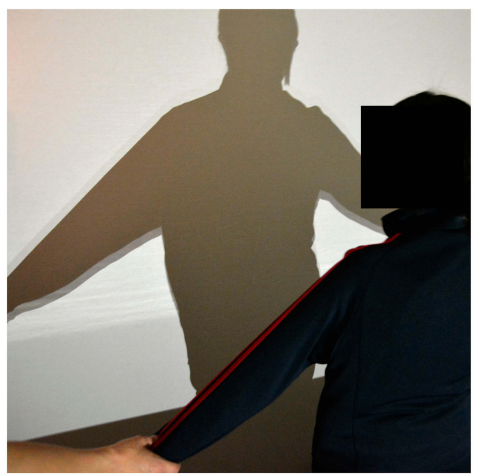

F

Figure I Body shadow methods. (A-C): Ist step, (D): 2nd step, (E): 3rd step, (F): shoulder adduction and abduction while looking at the body shadow. A cylindrical balloon was passed through the left sleeve of a long-sleeve shirt to imitate the patient's left arm (A). The patient wore the shirt but did not pass her left arm through the sleeve; her left hand was projected as a shadow using the therapist's left hand (B). When the body was projected, the patient repeatedly flexed and extended her right-hand fingers; the therapist also flexed and extended the fingers on his left hand in synchronization with the patient's hand (C). Consequently, the patient's motor intention and the visual feedback of the shadow formed an SoO and SoO over the body shadow of the left hand. Next, the imitated left arm was replaced with the patient's own left arm. Consequently, using her body shadow, the patient was able to "touch" her face (D) and another person's projected hand (E). Later, a body shadow of the patient's whole body was projected, and she performed adduction and abduction of both shoulders while looking at the body shadow (F).

\section{Results}

Table 1 shows the changes in symptoms and intervention methods.

\section{Changes in Pain}

On the first day of the body shadow intervention, the patient progressed to the first and then to the second step; as a result, the patient's pain rapidly decreased to NRS 6 and SF-MPQ-2 (total) 112. The patient continued to demonstrate alleviation of pain in the third body shadow session (day 7; NRS 3, SF-MPQ-2 [total] 79). Therefore, we transitioned to the third step of the intervention. By the 20th intervention session (day 48), the pain was at NRS 3 and SF-MPQ-2 (total) 54.

\section{Changes in Neglect-Like Symptoms}

The patient demonstrated improvements in NLS following commencement of the body shadow intervention. In the seventh body shadow session (day 13), the NLS score was 150 (MN, 110; CN, 40), which further improved to 125 $(\mathrm{MN}, 95 ; \mathrm{CN}, 30)$ in the $20^{\text {th }}$ body shadow session (day 48).

\section{Changes in Physical Self-Disgust and Autonomic Nervous Symptoms}

The patient's initial physical self-disgust rating of 10 decreased to 5 immediately after initiating the body shadow intervention and to 1 by the 20 th body shadow session (day 48), indicating near-complete disappearance of self-disgust. Similar results were observed for autonomic nervous symptoms; although the patient initially demonstrated marked left-right differences in autonomic nervous symptoms, she demonstrated improvement immediately after beginning the body shadow intervention. In the $20^{\text {th }}$ session (day 48 ), we observed no leftright differences. 


\section{Discussion}

In this case of CRPS, the patient presented with NLS accompanied by extensive pain primarily in her left arm, absence of a body image, and a physical self-disgust and repulsion toward her own limb. The patient's pain was exacerbated by the interrelationship between a sensorimotor representation associated with severe NLS and a metarepresentation of "No one would want to be touched with a hand like this, they'd be creeped out" formed from a conceptual representation where the patient described her affected limb as "reptilian" and stated, "I don't want to touch my face with this hand; I'm scared." Consequently, even after MT, the patient demonstrated no change in the physical self-disgust or repulsion upon seeing her actual left hand and was still afraid of touch. Therefore, we conducted a body shadow-based intervention, which led to a body image formation without physical self-disgust and enabled the patient to touch her own face and other people without fear or repulsion. The physical self-disgust in the present case may have been acquired, developing as a result of the fear and pain experienced. Body shadows are seen naturally on a daily basis, and because they represent the affected limb in an abstract manner, we believed that they can generate $\mathrm{SoO}$ and $\mathrm{SoA}$ without eliciting physical self-disgust and allow the patient to form a body image.

In the present case, the patient demonstrated physical self-disgust toward her own affected limb, complaining "it's thinner than it looks, and the color makes me sick; it looks like a reptilian limb." Feelings of physical selfdisgust and repulsion toward one's own body have been shown to exacerbate pain. ${ }^{27-29}$ Many people perceive the reptiles as repulsive. This is a conceptual representation; one's own concepts affect the level of pain felt and motor control at the sensorimotor level in a top-down fashion. Our patient also demonstrated repulsion in the meta-representation of her own body, stating, "I don't want to touch my face with this hand; I'm scared" and "No one else would want to be touched with a hand like this, either." Synofzik et $\mathrm{al}^{30}$ divided body awareness into three levels (sensorimotor representation, conceptual representation, and meta-representation) and explained that these three levels interact and affect each other. We interpreted the patient's pathology as follows: due to pain, the patient used her affected site less frequently, leading to NLS in sensorimotor representation; her symptoms were became complex by the interaction between this sensorimotor representation, a conceptual representation wherein the patient perceived her own limb to be reptilian, and a meta-representation consisting of the social awareness that no one else would want to be touched by her hand.

In the first step, we created an imitation of the patient's affected limb (the left arm). The patient remarked, "I feel like I'm moving this body shadow hand," indicating that she had formed an SoA. The SoA is considered to arise from the temporal synchronization and congruence of motor intention with feedback from somatosensory and visual information. ${ }^{31}$ The movement of the left hand in synchronization with the patient's right hand, which showed her motor intention, created consistency between the patient's motor intention and feedback from visual information. This consistency may have produced an SoA over the body shadow created by the imitation left arm. The patient also stated, "It's as if this body shadow is my own hand," confirming the SoO emergence over the body shadow. When projecting a hand shadow directly above a subject's hand, Kodaka et $\mathrm{al}^{32}$ found that their healthy subjects developed an $\mathrm{SoO}$ over the hand shadow merely by observing it and reported that body shadows can create body illusions as mirrors do. Synofzik et $\mathrm{al}^{30}$ reported that the sensation predicted to occur as a result of movement is formed in the brain as an efference copy and that SoA and $\mathrm{SoO}$ are formed when this prediction is consistent with the visual and somatosensory feedback generated when the movement is executed. This mechanism likely allowed the patient to form $\mathrm{SoO}$ and SoA over the imitated hand's body shadow. This formation of SoO and SoA may have also improved NLS and thus led to pain alleviation.

In the second step, we created body shadows using the patient's own hand instead of the imitation left arm. When she observed her own limb projected as a body shadow, she said, "I can sense that it's my hand, but it doesn't look reptilian. It doesn't creep me out," demonstrating that she had maintained an SoO without complaining of physical self-disgust. Later, using a body shadow of her own hand, the patient was able to actively "touch" (without actually touching) other people's hands, objects, and her own face. As reported by Pavani and Galfano ${ }^{24}$ and by Kuylen et al, ${ }^{33}$ bringing one's body shadow close to an object reduces the subjective distance from it, even if the object is not physically touched. This shows that a person can simulate touching objects without touching them, using a body shadow over which that person has an SoO. In the present case, when the patient brought her body shadow hand 
close to another person's hand, she remarked, "I feel like my hand is really touching, but I'm not afraid to touch because it doesn't hurt." This indicates that contact tasks using body shadows enabled the patient to simulate touching without the pain or fear she expected to feel.

Regarding the analgesic effect of illusions produced with virtual reality in a study of knee pain associated with knee osteoarthritis, Stanton et $\mathrm{al}^{34}$ reported that visual information synchronized with somatosensory information produced more potent illusions and a greater analgesic effect than visual information alone did. In the present case, the illusions of contact sensation produced through body shadows synchronized with the visual information of the patient touching others with the body shadow of her own limb, resulting in more potent body illusions. This finding indicates that body shadows not only served as simulations of touching but also yielded an analgesic effect. Thus, interventions with body shadows are considered significant for two reasons: they can be used to simulate touch, which is not possible with imaged movement or MT, and they are introduced after MT as a preparatory step before actual contact tasks.

In the third step, we projected a body shadow of the patient's entire body and performed adduction and abduction of an imitation left arm in synchronization with the patient's intention as demonstrated by her right arm, thereby forming SoO and SoA over her left shoulder and trunk. This SoO and SoA were maintained even after the imitation left arm was replaced with the patient's own left arm. Body shadows did not trigger physical self-disgust from the patient to any part of her left arm and alleviated pain during shoulder movement. These effects may have been responsible for reducing fear and the expansion of shoulder ROM.

Autonomic nervous symptoms (skin color change, cold sensation, and edema) demonstrated the same course of improvement from the first step to the third step of body shadow intervention as pain, NLS, and physical self-disgust toward the affected limb. Pain and the fear of pain have been shown to be involved in swelling and other autonomic nervous symptoms, ${ }^{35}$ while seeing the affected limb as bigger than normal has been reported to exacerbate autonomic nervous symptoms; ${ }^{36}$ these findings suggest that awareness of one's own limb affects autonomic nervous symptoms. In the present case, the patient's physical self-disgust was also affected by the unpleasant color extending from her left forearm to her fingers. The use of body shadows led to an SoO over the affected limb without physical self-disgust, which improved NLS and alleviated pain; these effects may have improved autonomic nervous symptoms.

A limitation of the present intervention is that it assessed a single case; thus, we cannot rule out the effect of spontaneous recovery. However, a control group would be difficult to establish, making it impossible to fully demonstrate the efficacy of body shadows. Furthermore, we did not assess brain activity or perform any other objective assessments regarding the mechanism by which body shadows alleviate pain.

\section{Conclusion}

Through this case, we showed that body shadow interventions can be performed to produce potent body illusions with the following characteristics: (1) they allow the patient to readily form $\mathrm{SoO}$ and SoA; (2) they can simulate touch without eliciting fear; and (3) because body shadows are abstract projections of the affected limb, they do not trigger physical self-disgust or repulsion. To the best of our knowledge, this is the first report to demonstrate the effectiveness of body shadow intervention in a patient with CRPS. Body shadows can be easily projected using a light source and a wall. Therefore, body shadows are an effective intervention tool for patients with CRPS who present with impaired recognition, physical self-disgust, and fear of using their affected limb.

\section{Acknowledgments}

We would like to thank Editage for English language editing assistance. This study did not receive any funding support from external sources.

\section{Disclosure}

The authors have no conflicts of interest in this work.

\section{References}

1. Baron R, Schattschneider J, Binder A, Siebrecht D, Wasner G. Relation between sympathetic vasoconstrictor activity and pain and hyperalgesia in complex regional pain syndromes: a case-control study. Lancet. 2002;359(9318):1655-1660. doi:10.1016/S0140-6736 (02)08589-6

2. Jänig W, Baron R. Complex regional pain syndrome: mystery explained? Lancet Neurol. 2003;2(11):687-697. doi:10.1016/S14744422(03)00557-X

3. Pleger B, Tegenthoff $M$, Ragert $P$, et al. Sensorimotor returning in complex regional pain syndrome parallels pain reduction. Ann Neurol. 2005;57(3):425-429. doi:10.1002/ana.20394

4. Schwenkreis P, Maier C, Tegenthoff M. Functional imaging of central nervous system involvement in complex regional pain syndrome. AJNR Am J Neuroradiol. 2009;30(7):1279-1284. doi:10.3174/ajnr.A1 630 
5. Birklein F, Dimova V. Complex regional pain syndrome-up-to-date. Pain Rep. 2017;2(6):e624. doi:10.1097/PR9.0000000000000624

6. Di Pietro F, McAuley JH, Parkitny L, et al. Primary somatosensory cortex function in complex regional pain syndrome: a systematic review and meta-analysis. J Pain. 2013;14(10):1001-1018. doi:10.10 16/j.jpain.2013.04.001

7. Lenz M, Höffken O, Stude P, et al. Bilateral somatosensory cortex disinhibition in complex regional pain syndrome type I. Neurology. 2011;77(11):1096-1101. doi:10.1212/WNL.0b013e31822e1436

8. Maihöfner C, Handwerker HO, Neundorfer B, Birklein F. Patterns of cortical reorganization in complex regional pain syndrome. Neurology. 2003;61(12):1707-1715. doi:10.1212/01.WNL.00000989 $39.02752 .8 \mathrm{E}$

9. Juottonen K, Gockel M, Silén T, Hurri H, Hari R, Forss N. Altered central sensorimotor processing in patients with complex regional pain syndrome. Pain. 2002;98(3):315-323. doi:10.1016/S0304-3959 (02)00119-7

10. Cohen H, McCabe C, Harris N, Hall J, Lewis J, Blake DR. Clinical evidence of parietal cortex dysfunction and correlation with extent of allodynia in CRPS type 1. Eur J Pain. 2013;17(4):527-538. doi:10.1002/j.1532-2149.2012.00213.x

11. Lewis JS, Kersten P, McCabe CS, McPherson KM, Blake DR. Body perception disturbance: a contribution to pain in complex regional pain syndrome (CRPS). Pain. 2007;133(1):111-119. doi:10.1016/j. pain.2007.03.013

12. Vartiainen NV, Kirveskari E, Forss N. Central processing of tactile and nociceptive stimuli in complex regional pain syndrome. Clin Neurophysiol. 2008;119(10):2380-2388. doi:10.1016/j.clinph.2008. 06.008

13. Gieteling EW, van Rijn MA, de Jong BM, et al. Cerebral activation during motor imagery in complex regional pain syndrome type 1 with dystonia. Pain. 2008;134(3):302-309. doi:10.1016/j.pain.2007.04.029

14. McCabe CS, Haigh RC, Halligan PW, Blake DR. Simulating sensorymotor incongruence in healthy volunteers: implications for a cortical model of pain. Rheumatology (Oxford). 2005;44(4):509-516. doi:10. 1093/rheumatology/keh529

15. Swart CM, Stins JF, Beek PJ. Cortical changes in complex regional pain syndrome (CRPS). Eur J Pain. 2009;13(9):902-907. doi:10.10 16/j.ejpain.2008.11.010

16. van Hilten JJ. Movement disorders in complex regional pain syndrome. Pain Med. 2010;11(8):1274-1277. doi:10.1111/j.1526-4637. 2010.00916.x

17. Reinersmann A, Landwehrt J, Krumova EK, et al. The rubber hand illusion in complex regional pain syndrome: preserved ability to integrate a rubber hand indicates intact multisensory integration. Pain. 2013;154(9):1519-1527. doi:10.1016/j.pain.2013.03.039

18. Galer BS, Butler S, Jensen MP. Case reports and hypothesis: a neglect-like syndrome may be responsible for the motor disturbance in reflex sympathetic dystrophy (Complex Regional Pain Syndrome1). J Pain Symptom Manage. 1995;10(5):385-391. doi:10.1016/08853924(95)00061-3

19. Galer BS, Jensen M. Neglect-like symptoms in complex regional pain syndrome: results of a self-administered survey. $J$ Pain Symptom Manage. 1999;18(3):213-217. doi:10.1016/S0885-3924 (99)00076-7
20. Ramachandran VS, Rogers-Ramachandran D. Synaesthesia in phantom limbs induced with mirrors. Proc Biol Sci. 1996;263(1369):377386.

21. Moseley GL. Graded motor imagery for pathologic pain: a randomized controlled trial. Neurology. 2006;67(12):2129-2134. doi:10. 1212/01.wnl.0000249112.56935.32

22. Moseley GL. Is successful rehabilitation of complex regional pain syndrome due to sustained attention to the affected limb? A randomised clinical trial. Pain. 2005;114(1):54-61. doi:10.1016/j.pain.20 04.11.024

23. Bowering KJ, O'Connell NE, Tabor A, et al. The effects of graded motor imagery and its components on chronic pain: a systematic review and meta-analysis. J Pain. 2013;14(1):3-13. doi:10.1016/j. jpain.2012.09.007

24. Pavani F, Galfano G. The multisensory body revealed through its cast shadows. Front Psychol. 2015;6:666. doi:10.3389/fpsyg.2015.00666

25. Sartori L, Castiello U. Shadows in the mirror. NeuroReport. 2013;24 (2):63-67. doi:10.1097/WNR.0b013e32835c6e6a

26. Frettlöh J, Hüppe M, Maier C. Severity and specificity of neglect-like symptoms in patients with complex regional pain syndrome (CRPS) compared to chronic limb pain of other origins. Pain. 2006;124 (1):184-189. doi:10.1016/j.pain.2006.04.010

27. Sündermann O, Rydberg K, Linder L, Linton SJ. "When I feel the worst pain, I look like shit" - body image concerns in persistent pain. Scand J Pain. 2018;18(3):379-388. doi:10.1515/sjpain-2017-0163

28. Osumi M, Imai R, Ueta K, Nobusako S, Morioka S. Negative body image associated with changes in the visual body appearance increases pain perception. PLoS One. 2014;9(9):e107376. doi:10.13 71/journal.pone. 0107376

29. Osumi M, Imai R, Ueta K, Nakano H, Nobusako S, Morioka S. Factors associated with the modulation of pain by visual distortion of body size. Front Hum Neurosci. 2014;8:137. doi:10.3389/ fnhum.2014.00137

30. Synofzik M, Vosgerau G, Newen A. I move, therefore I am: a new theoretical framework to investigate agency and ownership. Conscious Cogn. 2008;17(2):411-424. doi:10.1016/j.concog.2008. 03.008

31. Haggard P, Clark S, Kalogeras J. Voluntary action and conscious awareness. Nat Neurosci. 2002;5(4):382-385. doi:10.1038/nn827

32. Kodaka K, Kanazawa A. Innocent body-shadow mimics physical body. I-Perception. 2017;8(3):2041669517706520. doi:10.1177/ 2041669517706520

33. Kuylen C, Balas B, Thomas LE. My shadow, myself: cast-body shadows are embodied. Psychon Bull Rev. 2014;21(3):676-681. doi:10.3758/s13423-013-0545-6

34. Stanton TR, Gilpin HR, Edwards L, Moseley GL, Newport R. Illusory resizing of the painful knee is analgesic in symptomatic knee osteoarthritis. PeerJ. 2018;6:e5206. doi:10.7717/peerj.5206

35. Moseley GL, Zalucki N, Birklein F, Marinus J, van Hilten JJ, Luomajoki $\mathrm{H}$. Thinking about movement hurts: the effect of motor imagery on pain and swelling in people with chronic arm pain. Arthritis Rheum. 2008;59(5):623-631. doi:10.1002/art.23580

36. Moseley GL, Parsons TJ, Spence C. Visual distortion of a limb modulates the pain and swelling evoked by movement. Curr Biol. 2008;18(22):R1047-8. doi:10.1016/j.cub.2008.09.031

\section{Publish your work in this journal}

The Journal of Pain Research is an international, peer reviewed, open access, online journal that welcomes laboratory and clinical findings in the fields of pain research and the prevention and management of pain. Original research, reviews, symposium reports, hypothesis formation and commentaries are all considered for publication. The manuscript

Submit your manuscript here: https://www.dovepress.com/journal-of-pain-research-journal management system is completely online and includes a very quick and fair peer-review system, which is all easy to use. Visit http:// www.dovepress.com/testimonials.php to read real quotes from published authors. 